\title{
Characterization of a DNA Aptamer for Ovarian Cancer Clinical Tissue Recognition and in Vivo Imaging
}

\author{
Fengjie Lia Qian Wang ${ }^{a}$ Hui Zhang ${ }^{b}$ Tanggang Deng ${ }^{b} \quad$ Peifu Feng ${ }^{b}$ \\ Bin $\mathrm{Hu}^{\mathrm{b}}$ Yanping Jiang ${ }^{\mathrm{a}}$ Lanqin $\mathrm{CaO}^{\mathrm{a}}$
}

${ }^{a}$ Xiangya Hospital, Central South University, Changsha, ' Molecular Science and Biomedicine Laboratory, State Key Laboratory for Chemo/Bio-Sensing and Chemometrics, College of Chemistry and Chemical Engineering, College of Biology, and Collaborative Research Center of Molecular Engineering for Theranostics, Hunan University, Changsha, China

\section{Key Words}

DNA aptamer • Ovarian cancer $•$ Membrane proteins $\bullet$ Endocytosis $\bullet$ Tissue microarray $\bullet$ Nude mouse

\begin{abstract}
Backgrounds/Aims: Ovarian cancer is the most lethal gynaecologic malignancy and is difficult to detect early. The inefficient early diagnosis of ovarian cancer is the main contributor to its high mortality rate. Aptamers, as chemical antibodies, are single-stranded DNA or RNA oligonucleotides that target cells or molecules with high affinity. Methods: Binding ability of R13 was measured by flow cytometry analysis. Stability of R13 was tested in blood serum of an ovarian cancer patient. Internalization of R13 was verified by confocal microscope imaging. 80 cases ovarian cancer tissues, 10 cases normal ovary tissues in a microarray and 6 fallopian tube tissues were prepared for this study. R13's target ability was further confirmed in vivo tumor models in NOD/SCID mice. Results: In this study, we found aptamer R13 bound to ovarian cancer cells with dissociation constants in the nanomolar range. Moreover, these results were further confirmed by tissue imaging. Next we demonstrated that the targets of R13 are membrane proteins and that its internalization occurs in a caveolae-mediated and clathrin-mediated manner. The target function of R13 was determined by imaging A2780 tumours in mouse models. Conclusion: These findings suggest that R13 is a promising novel tool to diagnose and deliver drugs to treat ovarian cancer.




\section{Introduction}

Ovarian cancer is the fifth leading cause of tumour-related deaths in the United States [1]. Because it is diagnosed in an advanced stage and frequently exhibits recurrence, the morbidity of ovarian cancer is high, and its incidence ranks third among gynaecologic tumours [2]. According to article, 22, 400 patients are diagnosed with and 14, 000 patients die from the disease each year [3]. Currently, neoadjuvant chemotherapy followed by debulking surgery is recognized as a superior option to primary debulking surgery. However, neither surgery nor neoadjuvant chemotherapy can effectively reduce the recurrence rate [4]. The effect of chemotherapy drugs is greatly reduced due to the development of drug resistance. CA125 and HE4 are the most specific early detection biomarkers for ovarian cancer. However, their use is controversial due to their lack of sensitivity [5]. Deficiencies in treatment and diagnosis renders the 5 years survival rate remains below $50 \%$ [6]. Therefore, more effective molecular probes and therapeutic methods are needed to detect and treat ovarian cancer.

More than a decade ago, a dualistic model of epithelial ovarian carcinogenesis was proposed. Compared to type I tumours (endometriosis-related tumours, low-grade serous carcinomas, mucinous carcinomas and malignant Brenner tumours), the morbidities of type II tumours (various high-grade serous carcinoma, carcinosarcoma, and undifferentiated carcinoma) are much higher due to their higher proliferative ability, ascites metastasis, the lack of effective early diagnosis and frequent chemoresistance, leading to poor overall clinical outcomes. High-grade serous ovarian cancer (HGSOC), a type of extremely malignant type II tumour, accompanied by extensive early metastasis, causes most of the morbidity and mortality associated with ovarian carcinomas [7]. Both clinical observations and mechanistic research support the hypothesis that the distal end of the tube is the main origin of early HGSOC carcinogenesis [8-12].

Aptamers are short, single-strand oligo(deoxy)nucleotides that fold into distinct tertiary structures and can be screened from a $10^{10}$ random single chain oligonucleotide (ssDNA or RNA) library and chemically synthesized by systematic evolution of ligands by exponential enrichment (SELEX) [13-14]. Aptamers bind to various target molecules, such as cells, viruses, proteins, and bacteria with high specificity [15]. This high specificity and selectivity make them ideal bioreceptors in aptasensors (aptamer-based biosensors). Several electrochemical, optical, and colorimetric aptasensor methods have been used to detect cancers [16]. At present, many aptamers have been applied in biomedical research for cancer cell detection, cell capture, imaging, targeted therapy and biomarker discovery. These molecules show potential for application in early cancer diagnosis and targeted therapeutics [17-19]. Though similar to antibodies, aptamers have several attractive advantages over antibodies, including a smaller molecular weight, a shorter synthesis time, the ability to distinguish diverse targets, higher specificity and affinity, no or low immunogenicity, and long-term stability, which enable aptamer-delivered drugs to take effect without harming normal cells, and their production costs are lower than those of monoclonal antibodies [18]. Additionally, their stability makes aptamers superior to antibodies for detecting biomarkers, which is useful in the fight against cancer [20]. However, the SELEX process of aptamers is labourious and time-consuming, and the optimization of various steps in Cell-SELEX procedures is difficult to ensure [13]. In this study, we have found an existing aptamer, R13, that targets ovarian cancer cells. This aptamer was previously selected by Cell-SELEX against the A549 lung cancer cell line stably transfected with EGFR-GFP [21].

The R13 aptamer binds to ovarian cancer cells with particularly high affinity. Binding analysis revealed the strong affinities between R13 and A2780, H08910 and SKOV3 (kd values are all below 50nmol). The targeting ability of R13 was verified in tissues, and similar results were also observed in vivo. Therefore, R13 can recognize ovarian cancer. Furthermore, we showed that R13 targets extracellular membrane proteins and that it can be internalized into H08910 and A2780 cells through caveolae- and clathrin-mediated pathways. Therefore, R13 can be considered a promising tool for ovarian cancer diagnosis and treatment. 


\section{Cellular Physiology Cell Physiol Biochem 2018;51:2564-2574 and Biochemistry \begin{tabular}{l|l} 
DOI: 10.1159/000495925 & (c) 2018 The Author(s). Published by S. Karger AG, Basel \\
www.karger.com/cpb
\end{tabular}}

Li et al.: An DNA Aptamer Identify Ovarian Cancer

\section{Materials and Methods}

\section{Cell lines and culture}

The human ovarian cancer cell lines H08910 and Caov3 were purchased from the Type Culture Collection of the Chinese Academy of Sciences (Shanghai, China). A2780 and SKOV3 cells were were obtained from the American Type Culture Collection (ATCC, Manassas, VA). H08910 and Caov3 cells were cultured in RPMI-1640 medium supplemented with 10\% foetal bovine serum (FBS, Zeta) and $100 \mathrm{U} / \mathrm{ml}$ penicillinstreptomycin. The A2780 cells were cultured in DMEM medium supplemented with 10\% FBS (Zeta) and $100 \mathrm{U} / \mathrm{ml}$ penicillin-streptomycin. The SKOV3 cells were cultured in Maccoy5A medium supplemented with $10 \%$ FBS (Zeta), and all the cell lines were cultured at $37^{\circ} \mathrm{C}$ in a humid atmosphere with $5 \% \mathrm{CO}_{2}$.

\section{Buffer and reagents}

Washing buffer was prepared with $500 \mathrm{ml}$ of Dulbecco's phosphate-buffered saline (DPBS, Gibicol, USA), $5 \mathrm{mM} \mathrm{MgCl} 2$ and $2.25 \mathrm{~g}$ glucose at a final concentration of $4.5 \mathrm{mg} / \mathrm{ml}$. Washing buffer was stored at $4^{\circ} \mathrm{C}$ (used within 2 months). The binding buffer used in the experiments consisted of washing buffer containing $0.1 \mathrm{mg} / \mathrm{ml}$ of yeast tRNA (Sigma) and $1 \mathrm{mg} / \mathrm{l}$ of BSA. Briefly, $0.2 \%$ EDTA ( $0.1 \mathrm{~g} \mathrm{Na}_{2}$ EDTA powder) was diluted in $50 \mathrm{ml}$ of DPBS. Blocking buffer (20\% FBS and $0.1 \mathrm{mg} / \mathrm{ml}$ salmon sperm DNA) was added to the binding buffer. The antigen repair solution consisted of $0.01 \mathrm{M}$ sodium citrate buffer, $\mathrm{pH}=6.0$. The inhibitors dynasore and genistein were purchased from Selleckchem.com, and amiloride was obtained from MedChemExpress (MCE).

\section{DNA synthesis and labelling}

All the aptamers were synthesized by Sangon Biotech Co., Ltd. (Shanghai, China). The sequence of R13 is TCTCTAGTTATTGAGTTTTCTTTTATGGGTGGGTGGGGGGTTTTT. The ssDNA control aptamer consisted of a random sequence of 45 bases (NNNNNNNNNNNNNNNNNNNNNNNNNNNNNNNNNNNNNNNNNNNNN), and FAM and Cy5 were used to label the 5' terminal of the DNA.

\section{Flow cytometry}

When cells were grown to $80 \%$ confluency, they were detached by adding $0.02 \%$ EDTA for 3-5 min. After rinsing with washing buffer, approximately $3 \times 10^{5}$ cells were collected and counted. Following the incubation of cells with $250 \mathrm{nM}$ R13 labelled with FAM for $40 \mathrm{~min}$, nonspecific binding was reduced by washing the cells twice. After filtration with $500 \mu \mathrm{l}$ of washing buffer, the binding ability was measured with a BD FACSVerse ${ }^{\mathrm{TM}}$ flow cytometer. All the binding ability experiments were replicated more than 3 times.

\section{Affinity analysis}

Based on the flow cytometry assay, we investigated the equilibrium dissociation constants $\left(\mathrm{K}_{\mathrm{d}}\right)$ by incubating cancer cells with different concentrations of R13 and then analysed the mean florescence intensity by flow cytometry, according to the equation $\mathrm{Y}=\mathrm{B}_{\max } \mathrm{X} /\left(\mathrm{K}_{\mathrm{d}}+\mathrm{X}\right)(\mathrm{X}$ indicates the concentration, $\mathrm{Y}$ represents the dependence of the fluorescence intensity on the aptamer/cell complex). We calculated the $\mathrm{K}_{d}$ values of R13 using GraphPad Prism5.

\section{Differential combination with tissues}

The OV809 tissue microarray model was purchased from Xi'an Alena Biological Technology Co., Ltd. (China). Six distal fallopian tube tissues were collected at the Department of Pathology of Xiangya Hospital (Changsha, China). The tissues were obtained from patients who underwent surgeries due to benign ovarian lesions. After incubation in an oven at $60^{\circ} \mathrm{C}$ for 2 hours, the tissue slices were deparaffinized by immersion in xylene for $15 \mathrm{~min}$. Hydration of the sections was performed by immersing the slices into decreasing concentrations of ethanol (100\%, 95\%, 90\%, 80\%, and 70\%) for $5 \mathrm{~min}$ each. Then, the tissue sections were immersed in $0.01 \mathrm{M}$ citrate buffer $(\mathrm{pH}=6.0)$, and the buffer was heated to $95^{\circ} \mathrm{C}$ in a sterilizer; $20 \mathrm{~min}$ later, the sections were removed from the sterilizer. The slices were cooled by washing in ultra-pure water and then incubated with blocking buffer for 1 hour on a low-speed shaker. After rinsing, the tissues were incubated with $250 \mathrm{nM}$ Cy5-labelled R13 for 1 hour. After 3 washes with DPBS for 10 min for each, the sections were sealed. After the resin dried, fluorescence images were obtained with a Pannoramic MIDI II 


\section{Cellular Physiology Cell Physiol Biochem 2018;51:2564-2574 \begin{tabular}{ll|l} 
and Biochemistry Published onlIne: 11 December 2018 & $\begin{array}{l}\text { (c) } 2018 \text { The Author(s). Published by S. Karger AG, Basel } \\
\text { www.karger.com/cpb }\end{array}$ \\
\hline
\end{tabular}}

Li et al.: An DNA Aptamer Identify Ovarian Cancer

slide scanner, and the fluorescence intensity was calculated with Quant Center. The signal intensity scale ranged from $6 \times 10^{4}$ to $10 \times 10^{6}$. Finally, statistical analysis was performed based on the specific fluorescence values.

\section{Confocal microscopic imaging}

The cells were grown in a glass-bottom cell culture dish for at least 24 hours; when the density reached $60 \%$, the cells were washed twice with precooled washing buffer and then incubated with 250 nmol of aptamers labelled with FITC or Cy5 for $40 \mathrm{~min}$ at $37^{\circ} \mathrm{C}$ or $4^{\circ} \mathrm{C}$. The cells were then washed 3 times to eliminate nonspecific binding. Images were obtained with an FV1000-X81 confocal microscope (Olympus, Japan), and FV10-ASW Version 3.1 software was used to analyse the images [22].

\section{Endocytosis pathway analysis}

To determine the pathway of R13 internalization, we preincubated cells with $80 \mu$ mol dynasore (inhibitor of clathrin-mediated endocytosis) [23], $50 \mu \mathrm{mol}$ genistein (inhibitor of caveolae-mediated endocytosis) [24], or $50 \mu \mathrm{mol}$ amiloride (inhibitor of macropinocytosis) for $30 \mathrm{~min}$ at $37^{\circ} \mathrm{C} \mathrm{[25].} \mathrm{Then,} \mathrm{the}$ cells were incubated with aptamers for $40 \mathrm{~min}$ at $37^{\circ} \mathrm{C}$. Immediately after 3 washes, images were obtained with an FV1000-X81 confocal microscope (Olympus, Japan) [26].

\section{Target type analysis}

To determine whether the targets of R13 are membrane proteins, the cells were treated with $0.25 \%$ trypsin or $0.1 \mathrm{mg} / \mathrm{ml}$ protease $\mathrm{K}$ to damage the membrane proteins before incubation with R13. Then, changes in flow cytometry assays were detected.

\section{Serum stability assay}

We determined the stability of R13 in human serum obtained from a preoperative ovarian cancer patient in ward 18, Xiangya Hospital. To achieve a final concentration of $3 \mu \mathrm{M}, 55.8 \mu \mathrm{l}$ of R13 was added (at a concentration of $10 \mu \mathrm{mol}$ ) to $117.2 \mu \mathrm{l}$ of RPMI 1640 medium containing $13 \mu \mathrm{l}$ human serum. The samples were incubated for up to 24 hours at $37^{\circ} \mathrm{C}$, and samples were collected at certain time points $(0,0.5,1,3,6$, 12 , and 24 hours). The samples were heated to $100^{\circ} \mathrm{C}$ for $10 \mathrm{~min}$ and immediately stored at $-80^{\circ} \mathrm{C}$. Then, the stability was analysed by electrophoresis on a $3 \%$ agarose gel.

\section{Nude mouse xenograft tumour models}

We choose A2780 cells to establish xenograft tumour models due to their good tumourigenicity [27] and the high affinity of R13. Female athymic nude mice ( 5 weeks, 18-20 g) were purchased from Shanghai SLAC Laboratory Animal Co., Ltd. (Changsha, China). Approximately $1.5 \times 10^{7} / \mathrm{ml}$ of the A2780 cells were resuspended in $0.2 \mathrm{ml}$ of DPBS; then the tumour cell suspension was injected into the right shoulders of the mice. Approximately 15 days later, the tumour models were ready for use in experiments. We ensured that all the operations involving animals strictly abided by international ethics regulations. All the surgical operations were performed in an anaesthetized state.

\section{Tumour imaging in vivo via a multimode imaging system}

We injected the tail veins of two nude mice with $100 \mu \mathrm{l} \mathrm{R} 13$ or a control aptamer labelled with cy5' $(100$ $\mu \mathrm{l})$ and then obtained whole body fluorescence images at 0.5, 1.5, 3.5, 5.5, 7.5, 9.5, 10.5 and 11.5 hours with Living Image@4.3.1 (Lumina xr). After whole-body fluorescence images were obtained, we reinjected the mice with $100 \mu \mathrm{l}$ of R13 or the control aptamer. Then, the mice were sacrificed, and images of several organs (heart, spleen, lungs, liver and kidneys) were obtained half an hour later. 


\section{Results}

R13 binds to ovarian cancer cells

We tested the ability of R13 to bind to ovarian cancer cells by flow cytometry analysis. As shown in Fig. 1A, R13 binds to several ovarian cancer cell lines (A2780, H08910, SKOV3, and Caov3). Next, we quantified the binding affinity of R13 to ovarian cancer cells by measuring the equilibrium dissociation constants (Fig. 1B). The $\mathrm{K}_{\mathrm{d}}$ values were $\mathrm{K}_{\mathrm{d}}(\mathrm{HO8910})$ $=47.48 \pm 7.15, \mathrm{~K}_{\mathrm{d}}(\mathrm{A} 2780)=$ $29.24 \pm 8.55, \mathrm{~K}_{\mathrm{d}}(\mathrm{SKOV} 3)=$ $37.87 \pm 4.93$, and $\mathrm{K}_{\mathrm{d}}(\mathrm{Caov} 3)=$ $158 \pm 28.22$. Relative to ovarian adenocarcinoma cells in situ (Caov3), R13 has high affinities for H08910, A2780, and SKOV3, which indicates that R13 may exhibit stronger binding with malignant cancer cells.

\section{R13 has good targeting} ability in tissue sections

The targeting ability of R13 was demonstrated in a tissue array composed of 10 normal ovaries and 70 ovarian cancers of different pathological types and various stages. As shown in Table 1, approximately $80 \%$ of normal tissues showed negative fluorescence intensity, while all stage I, $85 \%$ of stage II, $80 \%$ of stage III, and $86 \%$ of stage IV cancer tissues showed different fluorescence signal levels, and we made a statistic analysis of the mean fluorescence intensity of normal ovary and ovarian cancer tissues at different stages (Fig. 2C), the $\rho$ value was below 0.05 , which provided evidence that R13 has the capability to distinguish ovarian cancer from normal ovaries. Simultaneously, we collected six distal ends of fallopian tubes resected from patients with benign cysts of the ovary or other benign lesions in Xiangya Hospital and tested the binding ability of R13. The fallopian tubes displayed much weaker fluorescence than did the HGSOC tissues of OV809 (Fig. 2B), indicating that R13 has the potential to identify HGSOC when the lesion originates in the fallopian tube.

\section{Secondary structure and stability of $R 13$}

The secondary structure of R13 (Fig. 3A) was predicted by Integrated DNA technologies. The sequence of R13 was predicted to have a large stem loop. We detected the stability of R13 in 10\% of the ovarian cancer patient serum samples (obtained from Xiangya Hospital). Slight degradation was detected after incubating $3 \mu \mathrm{mol} \mathrm{R} 13$ in serum for 24 hours at $37^{\circ} \mathrm{C}$ (Fig. 3B). 


\section{Cellular Physiology Cell Physiol Biochem 2018;51:2564-2574 and Biochemistry \begin{tabular}{l|l} 
DOI: 10.1159/000495925 2018 The Author(s). Published by S. Karger AG, Basel \\
(c) 2018 .
\end{tabular}

R13 targets the membrane proteins of cancer cells

We examined changes in R13 affinity after $0.25 \%$ trypsin or $0.1 \mathrm{mg} / \mathrm{ml}$ proteinase $\mathrm{K}$ was used to damage the membrane proteins of A2780 and H08910 cells [28]. As shown in Fig. 4, only a slight shift in affinity was observed after pretreatment with these compounds compared to the obvious shift observed after treatment of cells with $0.2 \%$ EDTA, which does not damage membrane proteins (Fig. 4), indicating that R13 likely targets extramembrane proteins.

Temperature-dependent internalization and endocytosis pathways of R13

We examined the internalization of R13 using an FV1000-X81 confocal microscope (Olympus, Japan). Images of A2780 and H08910 cells incubated with R13 labelled with Cy5, light microscopy images and merged images are shown in Fig. 5A and Fig. 5B. R13 is distributed only in the cell membranes when incubated with cells at $4^{\circ} \mathrm{C}$, but when the temperature was $37^{\circ} \mathrm{C}$, R13 was internalized into H08910 and A2780 cells to varying degrees. The time-dependent internalization of R13 indicates an active transport process (endocytosis). Endocytosis is a complex process that occurs via receptor-mediated pathways (clathrin-mediated, caveolaemediated, macropinocytosis and clathrinand caveolae-independent pathways) [29]; Therefore, we used inhibitors to

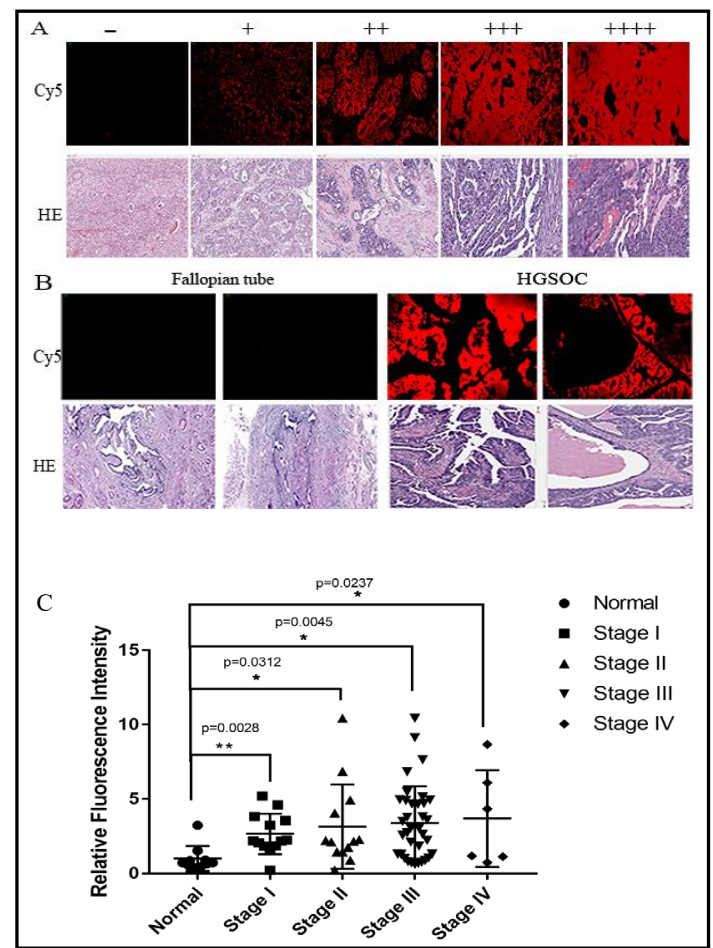

Fig. 2. Fluorescence and $H \& E$ images of tissues stained with R13 labelled with Cy5. (A) Typical fluorescence images of ovarian cancer cells stained with R13 labelled with Cy5 and images of H\&E staining of tissues. (B) Two typical cases of distal fallopian tube tissues stained with $H \& E$ and fluorescence images of serial sections of the same tissue. H\&E stained HGSOC slices and fluorescence images of HGSOC tissues are shown. (C) Summary statistics of the relative fluorescence intensity of normal ovary and ovarian cancer tissues at different stages.

Fig. 3. Secondary structure and stability of the R13 aptamer (A) Secondary structure of R13 predicted by Integrated Device Technology. (B) $\mathrm{R} 13(3 \mu \mathrm{mol})$ was incubated in $10 \%$ serum from an ovarian cancer patient for 24 hours; the stability was detected by $3 \%$ agarose gel electrophoresis.

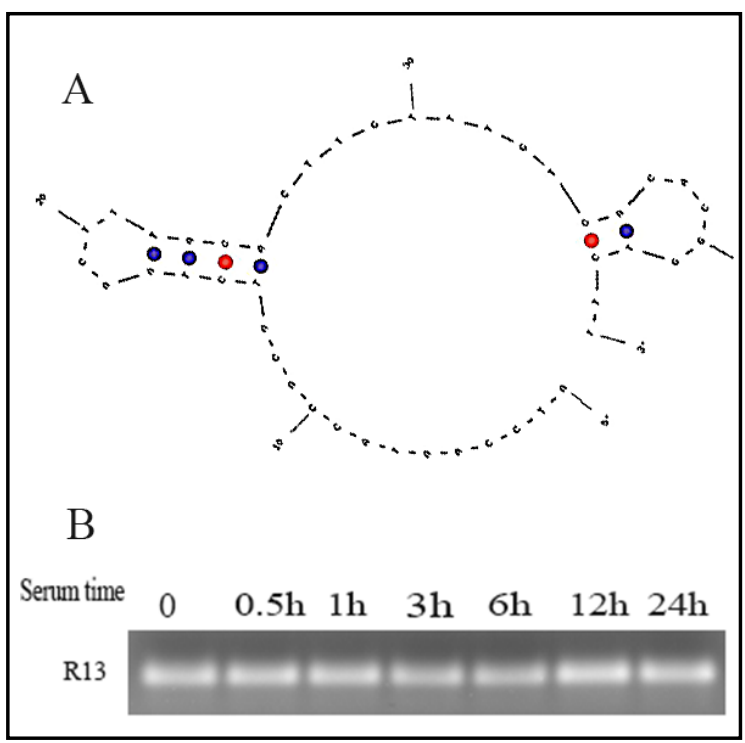


determine the endocytosis pathways of R13. We observed that the internalization of R13 decreased significantly (Fig. 6) after incubating cells with $80 \mu \mathrm{M}$ dynasore (inhibition of clathrin-mediated endocytosis) or $50 \mu \mathrm{M}$ genistein (inhibition of caveolin-mediated endocytosis) for $30 \mathrm{~min}$ at $37^{\circ} \mathrm{C}$, but when R13 was incubated with amiloride (inhibitor of the macropinocytosis pathway), it was internalized into cancer cells. Therefore, the uptake of R13 occurs by clathrin- and caveolae-mediated pathways.

\section{R13 targets tumours in nude mouse xenograft models}

To further investigate the recognition ability of R13 in vivo, nude mice injected with R13 labelled with Cy5 $(100 \mu \mathrm{M})$ were imaged using Living Image@4.3.1 (Lumina xr). A weak R13 signal emerged during the first 1.5 hours. As shown in Fig. 7A, an increase in the fluorescence signal occurred at 3.5 hours; this increase was still present at the 9.5th hour (Fig. 7, upper). No fluorescence signal was detected during the procedure after a library labelled with Cy5 $(100 \mu \mathrm{M})$ was injected into the mice (Fig. 7, lower). After $100 \mu \mathrm{l}$ of R13 or library was injected into mice through the tail vein, the mice were sacrificed, and the aptamers were imaged in tumours and organs half an hour later. The distribution of R13 and the control aptamer was detected, and fluorescence images of tumours, hearts, lungs, spleens, livers and kidneys are shown in Fig. 7B. The red fluorescence in the tumours of mice injected with R13 was

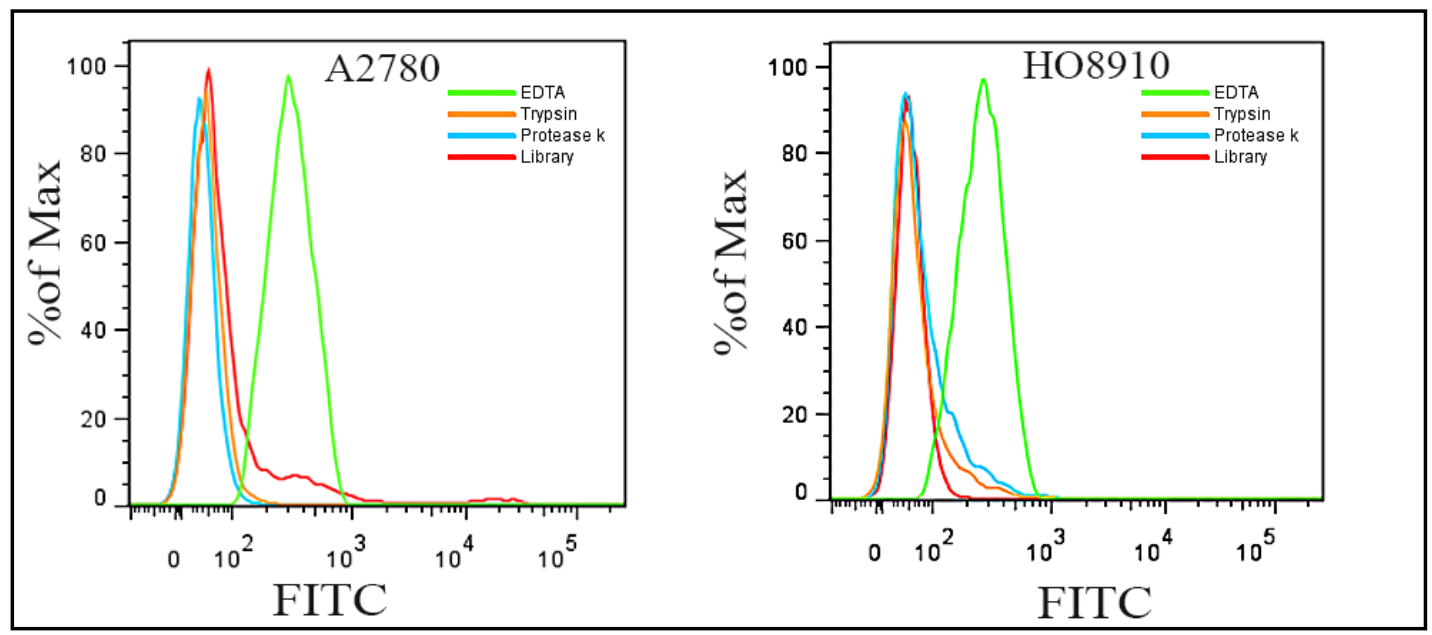

Fig. 4. Target type analysis of the R13 aptamer. Both H08910 and A2780 cells were treated with trypsin or protease $\mathrm{K}$ to damage membrane proteins before incubation with R13. R13 did not bind with pretreated cancer cells, demonstrating that the targets are likely membrane proteins.

Fig. 5. Internalization of the R13 aptamer in A2780 and H08910 cells. (A) Fluorescence, light microscopy and overlay images show the internalization of R13 into $\mathrm{A} 2780$ cells at $4^{\circ} \mathrm{C}$ or $37^{\circ} \mathrm{C}$. (B) Images of internalization of $\mathrm{R} 13$ into $\mathrm{H08910}$ cells at $4^{\circ} \mathrm{C}$ or $37^{\circ} \mathrm{C}$.

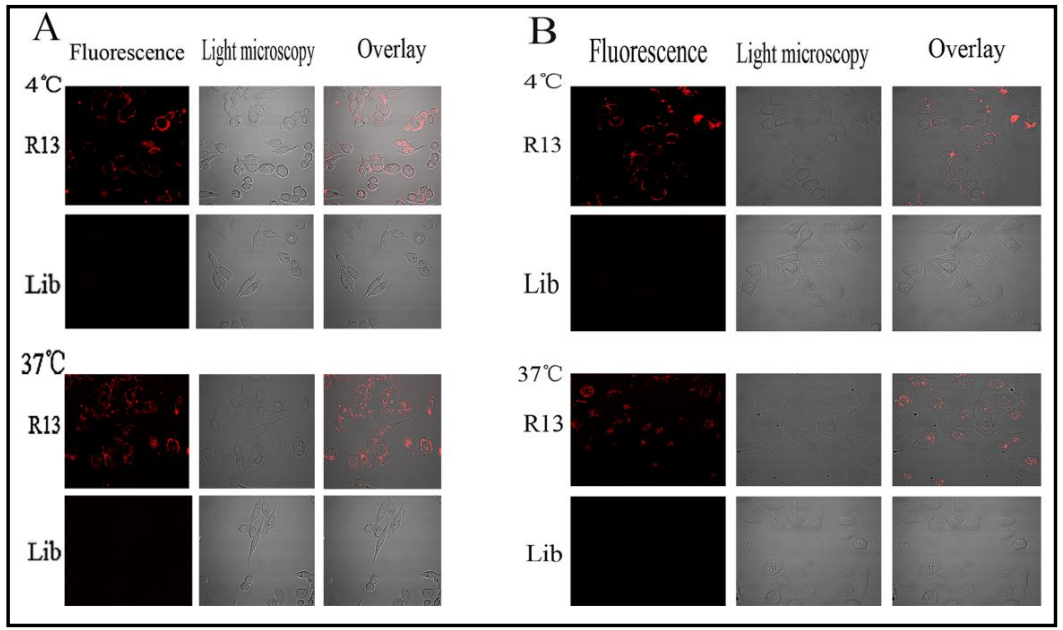




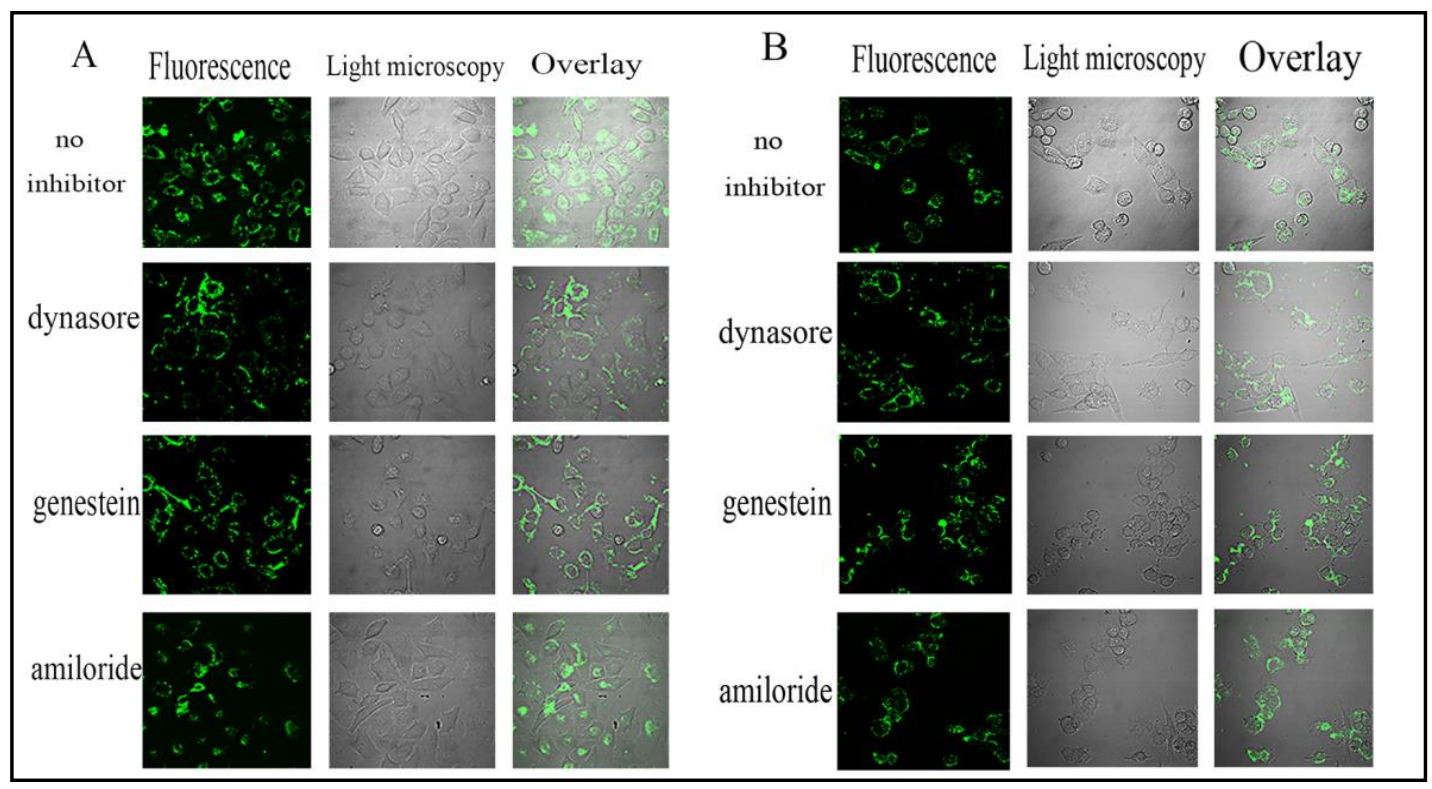

Fig. 6. Endocytosis pathway analysis of the R13 aptamer in A2780 and H08910 cells. (A) Fluorescence, light microscopy and overlay images show the endocytosis pathways of R13 in H08910 cells. (B) Images of endocytosis pathways of R13 in A2780 cells.

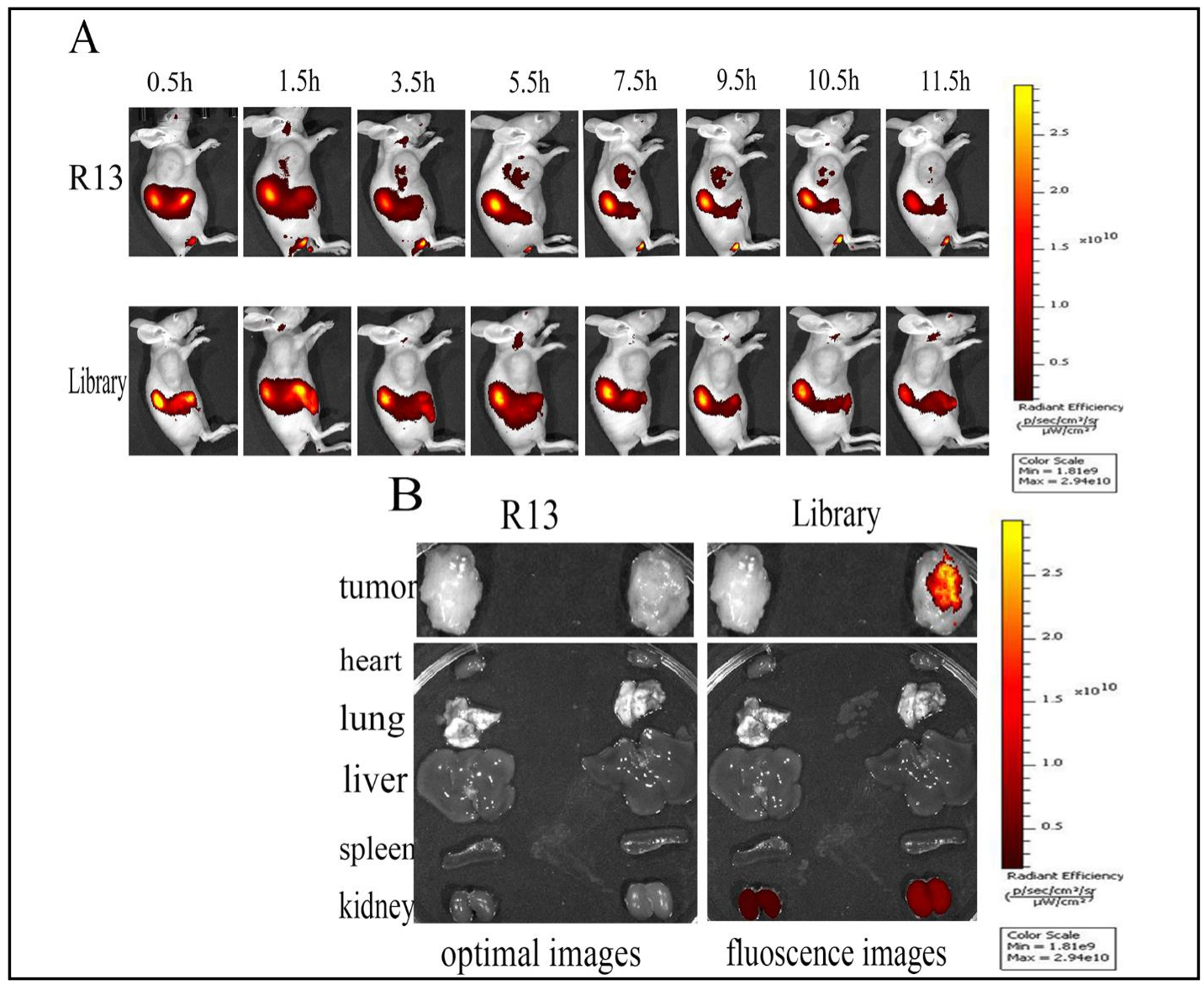

Fig. 7. In vivo imaging of the R13 aptamer or control aptamer labelled with Cy5. (A) Time-lapse fluorescence images of mice injected with R13 or the control aptamer (B) Fluorescence images of the hearts, lungs, livers, spleens and kidneys. 


\section{Cellular Physiology Cell Physiol Biochem 2018;51:2564-2574

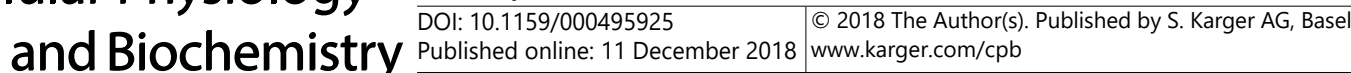 \\ Li et al.: An DNA Aptamer Identify Ovarian Cancer}

increased. Red signals in the lungs, hearts and spleens faded in 6 hours, and signals were not distributed to these organs at half an hour after another injection. However, strong signals were detected from the livers and kidneys.

\section{Discussion}

Aptamers, as single-stranded DNA or RNA with unique tertiary structures, can be uptaken in target cells quickly [30]. For example, DML-7, an DNA aptamer, binds with prostate cancer (PC) tissue specimens [19]. QD-A32 Apt showed breast cancer-specific accumulation in engrafted mice [31]. However, up to now, there are few studies investigating the use of fluorescent-labeled or gold metal-conjugated aptamers to assess cancer characteristics. Fortunately, we found an aptamer (R13) binds to ovarian cancer cells in vitro and in vivo and internalizes into cells through an endocytosis-mediated mechanism.

Ovarian cancer is the leading cause of death due to gynecological malignancy, and the current paradigms for early diagnosis and treatment are largely ineffective. In this article, we noticed R13 aptamer binds to ovarian cancer cells with Kd values in the nanomolar range. The Kd values revealed that R13 has strong affinities for A2780, H08910 and SKOV3. We further demonstrated that R13 has excellent selectivity for different tissues; its affinity for ovarian cancer tissues is apparently greater than that for normal ovarian tissues. High specifility revealed that R13 has potential for development into a novel tool for the diagnosis of and targeted drug delivery to malignant ovarian cells. Ovarian cancer xenograft model imaging also demonstrated the potential of R13 for the diagnosis and therapy of ovarian cancer. Additionally, R13 can distinguish between HGSOC and fallopian tube tissues, the deemed origin of HGSOC, which makes killing and detecting cancer cells in cradle possible.

We further showed that the targets of R13 are membrane proteins. These proteins are expressed on the surface of the tumour cells and can be classified as structural proteins, cell adhesion molecules, receptors, transporters or a combination of these, based on their functions [32]. Membrane proteins are highly accessible drug targets, in contrast to intracellular proteins and they may be used as biomarkers to distinguish cancer cells from normal cells [32]. Our current finding that R13 binds with discrepant ovarian cells and tissues differently arouse our interest to make clear what is/are the potential proteins R13 binds to?

Moreover, we discovered that R13 can be internalized into H08910 and A2780 cells at $37^{\circ} \mathrm{C}$ and that endocytosis occurs in a clathrin- and caveolin-mediated manner. As we all know, siRNA have great therapeutic potential and specificity, but one of their shortcomings remain the delivery and internalisation into cells [33]. R13 can enter cells specifically via cell mediated endocytosis, we envision potential problems of other oligonucleotide therapeutics such as siRNA, shRNA and miRNA can be solved. Furthermore, the answer that whether endocytosis increase in ovarian cancer compared to normal should be revealed? If the answer is yes, chemotherapy or radiotherapy agents, famous for their side effects, can be coupled to aptamers [34].

The high binding affinity and specificity for ovarian cancer and good stability, ability to bind to extracellular membrane proteins and internalization into cancer cells are advantageous for the development of future applications of this aptamer [35]. But an existing problem worries us: liver or kidney uptake can have nonspecific toxic effects. However, it's delightful that scientists are making every effort to reduce aptamer toxicity, for example, the relative distribution of the aptamer to the liver and kidney was found significantly decreased by hybridizing the aptamer to a photo-labile complementary oligonucleotide. 


\section{Cellular Physiology Cell Physiol Biochem 2018;51:2564-2574

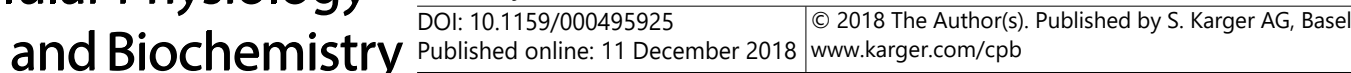

Li et al.: An DNA Aptamer Identify Ovarian Cancer

\section{Acknowledgements}

This study was supported by the Hunan Province Natural Science Key Fund Project (Grant 2017SK2071). L. C. designed and supervised the experiments; F. L. performed the experiments, analyzed the data and wrote the manuscript. H. Z. and T. D. gave technical support and conceptual advice. L. C., P. F, and H. B. gave critical advice to revise the manuscript. All authors read and approved the final manuscript.

\section{Disclosure Statement}

The authors declare that they have no competing interests.

\section{References}

1 Cornelison R, Llaneza D, Landen C: Emerging Therapeutics to Overcome Chemoresistance in Epithelial Ovarian Cancer: A Mini-Review. Int J Mol Sci 2017;DOI:10.3390/ijms18102171.

2 Langhe R: MicroRNA and Ovarian Cancer. Adv Exp Med Biol 2015;889:119-151.

3 Siegel R, Miller K, Jemal A: Cancer statistics, 2016. CA Cancer J Clin 2016;66:7-30.

4 Mukhopadhyay A, Bizzarri N, Bradbury M, Sinha S, Bhaumik J, Helm CW: Metastatic Involvement of Lesser Sac in Advanced Epithelial Ovarian Cancer. Int J Gynecol Cancer 2018;28:293-301.

5 Karakashev S, Aird K: Ovarian cancer: how can resistance to chemotherapy be tackled? Future Oncol 2017;13:2737-2739.

6 Runnebaum I, Reichert D, Ringsdorf U, Kuther M, Hesse T, Sehouli J, Wimberger P: Trabectedin plus pegylated liposomal doxorubicin (PLD) for patients with platinum-sensitive recurrent ovarian cancer: a prospective, observational, multicenter study. J Cancer Res Clin Oncol 2018;144:1185-1195.

7 Kroeger P, Drapkin R: Pathogenesis and heterogeneity of ovarian cancer. Curr Opin Obstet Gynecol 2017;29:26-34.

-8 Labidi Galy S, Labidi Galy SI, Papp E, Hallberg D, Niknafs N, Adleff V, Noe M, Bhattacharya R, Novak M, Jones S, Phallen J, Hruban CA, Hirsch MS, Lin DI, Schwartz L, Maire CL, Tille JC, Bowden M, Ayhan A, Wood LD et al.: High grade serous ovarian carcinomas originate in the fallopian tube. Nat Commun 2017;8:1093.

-9 Kurman R, Shih Ie M: The Dualistic Model of Ovarian Carcinogenesis: Revisited, Revised, and Expanded. Am J Pathol 2016;186:733-747.

10 Huang H, Hsu C, Chu S, Chen P, Ding D, Chang M, Chu T: Haemoglobin in pelvic fluid rescues Fallopian tube epithelial cells from reactive oxygen species stress and apoptosis. J Pathol 2016;240:484-494.

11 Perets R, Wyant G, Muto K, Bijron J, Poole B, Chin K, ChenJ, Ohman A, Stepule C, Kwak S, Karst A, Hirsch M, Setlur S, Crum C, Dinulescu D, Drapkin R: Transformation of the fallopian tube secretory epithelium leads to high-grade serous ovarian cancer in Brca;Tp53;Pten models. Cancer Cell 2013;24:751-765.

12 Wu N, Huang H, Chao T, Chou H, Fang C, Qin C, Lin C, Chu T, Zhou H: Progesterone Prevents High-Grade Serous Ovarian Cancer by Inducing Necroptosis of p53-Defective Fallopian Tube Epithelial Cells. Cell Rep 2017;18:2557-2565.

13 Rahimizadeh K, AlShamaileh H, Fratini M, Chakravarthy M, Stephen M, Shigdar S, Veedu RN: Development of Cell-Specific Aptamers: Recent Advances and Insight into the Selection Procedures. Molecules 2017; DOI:10.3390/molecules22122070.

14 Wang G, Liu J, Chen K, Xu Y, Liu B, Liao J, Zhu L, Hu X, Li J, Pu Y, Zhong W, Fu T, Liu H, Tan W: Selection and characterization of DNA aptamer against glucagon receptor by cell-SELEX. Sci Rep 2017;7:7179.

15 Mallikaratchy P, Tang Z, Kwame S, Meng L, Shangguan D, Tan W: Aptamer directly evolved from live cells recognizes membrane bound immunoglobin heavy mu chain in Burkitt's lymphoma cells. Mol Cell Proteomics 2007;6:2230-2238.

16 Li B, Chen C, Kumar U, Chen Y: Advances in nanowire transistors for biological analysis and cellular investigation. Analyst 2014;139:1589-1608. 


\section{Cellular Physiology Cell Physiol Biochem 2018;51:2564-2574 and Biochemistry \begin{tabular}{c|c} 
DOI: 10.1159/000495925 \\
Published
\end{tabular} \\ Li et al.: An DNA Aptamer Identify Ovarian Cancer}

17 Gold L: Oligonucleotides as research, diagnostic, and therapeutic agents. J Biol Chem 1995;270:1358113584.

18 Hori S, Herrera A, Rossi JJ, Zhou J: Current Advances in Aptamers for Cancer Diagnosis and Therapy. Cancers (Basel) 2018;DOI:10.3390/cancers10010009.

19 Duan M, Long Y, Yang C, Wu X, Sun Y, Li J, Hu X, Lin W, Han D, Zhao Y, Liu J, Ye M, Tan W: Selection and characterization of DNA aptamer for metastatic prostate cancer recognition and tissue imaging. Oncotarget 2016;7:36436-36446.

20 Wu X, Shaikh AB, Yu Y, Li Y, Ni S, Lu A, Zhang G: Potential Diagnostic and Therapeutic Applications of Oligonucleotide Aptamers in Breast Cancer. Int J Mol Sci 2017;DOI:10.3390/ijms18091851.

-21 Xu L, Zhang Z, Zhao Z, Liu Q, Tan W, Fang X: Cellular Internalization and Cytotoxicity of Aptamers Selected from Lung Cancer Cell. Am J Biomed Sci 2013;5:47-58.

-22 Jozefowski S, Srottek M: Lipid raft-dependent endocytosis negatively regulates responsiveness of J774 macrophage-like cells to LPS by down regulating the cell surface expression of LPS receptors. Cell Immunol 2017;312:42-50.

23 Imamura M, Kodama Y, Higuchi N, Kanda K, Nakagawa H, Muro T, Nakamura T, Kitahara T, Sasaki H: Ternary complex of plasmid DNA electrostatically assembled with polyamidoamine dendrimer and chondroitin sulfate for effective and secure gene delivery. Biol Pharm Bull 2014;37:552-559.

24 Koivusalo M, Welch C, Hayashi H, Scott CC, Kim M, Alexander T, Touret N, Hahn KM, Grinstein S: Amiloride inhibits macropinocytosis by lowering submembranous $\mathrm{pH}$ and preventing Rac1 and Cdc42 signaling. J Cell Biol 2010;188:547-563.

25 Li H, Yang S, Yu G, Shen L, Fan J, Xu L, Zhang H, Zhao N, Zeng Z, Hu T, Wen J, Zu Y: Aptamer Internalization via Endocytosis Inducing S-Phase Arrest and Priming Maver-1 Lymphoma Cells for Cytarabine Chemotherapy. Theranostics 2017;7:1204-1213.

-26 Xiao J, Xu M, Hou T, Huang Y, Yang C, Li J: Dasatinib enhances antitumor activity of paclitaxel in ovarian cancer through Src signaling. Mol Med Rep 2015;12:3249-3256.

27 Sui H, Shi C, Yan Z, Li H: Combination of erlotinib and a PARP inhibitor inhibits growth of A2780 tumor xenografts due to increased autophagy. Drug Des Devel Ther 2015;9:3183-3190.

28 Xu J, Teng IT, Zhang L, Delgado S, Champanhac C, Cansiz S, Wu C, Shan H, Tan W: Molecular Recognition of Human Liver Cancer Cells Using DNA Aptamers Generated via Cell-SELEX. PLoS One 2015; DOI:10.1371/ journal.pone.0125863.

29 Gao H, Yang Z, Zhang S, Pang Z, Jiang X: Internalization and subcellular fate of aptamer and peptide dualfunctioned nanoparticles. J Drug Target 2014;22:450-495.

30 Yoon S, Rossi J: Targeted Molecular Imaging Using Aptamers in Cancer. Pharmaceuticals (Basel) 2018; DOI:10.3390/ph11030071.

31 Kim M, Jeong H, Kang S, Choi M, You Y, Im C, Lee T, Song I, Lee C, Rhee K, Lee Y, Park Y: Cancer-targeted Nucleic Acid Delivery and Quantum Dot Imaging Using EGF Receptor Aptamer-conjugated Lipid Nanoparticles. Sci Rep 2017;7:9474.

-32 Dua P, Kim S, Lee D: Nucleic acid aptamers targeting cell-surface proteins. Methods 2011;54:215-225.

-33 de Almeida C, Alves L, Rocha H, Cabral Neto J, Missailidis S: Aptamer delivery of siRNA, radiopharmaceutics and chemotherapy agents in cancer. Int J Pharm 2017;525:334-342.

-34 Li L, Tong R, Chu H, Wang W, Langer R, Kohane DS: Aptamer photoregulation in vivo. Proc Natl Acad Sci U S A 2014;111:17099-17103.

-35 Liu J, You M, Pu Y, Liu H, Ye M, Tan W: Recent developments in protein and cell-targeted aptamer selection and applications. Curr Med Chem 2011;18:4117-4125. 